\title{
Iterative Correlation-Based Controller Tuning: Frequency-Domain Analysis
}

\author{
A. Karimi, L. Mišković and D. Bonvin \\ Laboratoire d'Automatique, Ecole Polytechnique Fédérale de Lausanne, \\ CH-1015 Lausanne, Switzerland. e-mail: alireza.karimi@epfl.ch
}

\begin{abstract}
The correlation approach is considered for the model-following problem using closed-loop noisy data. The idea is to tune the controller parameters such that the output error between the real closed-loop system and the designed one be uncorrelated with the reference signal. This way, the controller parameters will not be influenced by noise. Since perfect decorrelation is not always possible, the twonorm of the cross-correlation function between the closed-loop output error and an instrumental variable vector is minimized iteratively using the stochastic approximation method. A frequency-domain interpretation of the criterion is given for a simple choice of the instrumental variables. The analysis shows that the noise has asymptotically no effect on the controller parameters. It is shown that the tuned controller compensates for the unmodeled dynamics, so that the designed performances for the initial model are satisfied for the real system as well. The theoretical results are illustrated via a simulation example.
\end{abstract}

Keywords: Controller tuning, iterative methods, correlation approach, frequency analysis.

\section{Introduction}

Data-driven control design seems to be a promising approach for tuning restricted-order controllers without or with only little use of models. In Model Reference Adaptive Control (MRAC) and Self-Tuning Regulation (STR) [1], the controller parameters are tuned to minimize the two-norm of the output error between the closed-loop system and a reference model. These approaches can be extended to the minimization of a general quadratic criterion using the Gauss-Newton iterative formula. In [8], the gradient of the criterion is computed using an on-line estimated model of the plant, while in the Iterative Feedback Tuning (IFT) approach [2] the gradient is estimated using the closed-loop data without any model of the plant. Although model following is usually one of the objectives of the iterative controller tuning methods, it is not considered as the main objective. It is well known that, in the presence of noise and a penalty on the plant input, there is a trade-off between model following, noise attenuation and control signal limitation [1].

Recently, a new approach to model following based on the correlation technique has been proposed in $[3,4]$. The main idea is to make the output error between the designed 
and achieved closed-loop systems uncorrelated with the reference signal. The idea can be motivated as follows. Let a reduced-order model of the plant be available and an initial controller based on this model be designed so that all closed-loop specifications are satisfied. If this controller is implemented on the real system, performance degradation will occur because of differences between the reduced-order model and the real plant. Since the closed-loop output error contains the modeling errors (correlated with the reference signal) and noise, the controller should be tuned to compensate for the unmodeled plant dynamics. This way, the closed-loop output error contains only the contribution of noise, that is uncorrelated with the reference signal. It should be noted that the designed closed-loop system is more complex than a simple reference model for tracking since it represents a closed-loop system that meets all the specifications for tracking, control signal limitation, disturbance and noise attenuation for the initial model. Therefore, in this context, there is no interest in attenuating the output noise more than planned in the designed closed-loop system with the risk of missing the model matching performance. Hence, the objective is to make the achieved closed-loop system as close as possible to the designed one, independent of the noise characteristics.

The controller parameters in this approach are solutions to a correlation equation involving instrumental variables computed iteratively using the Newton-Raphson algorithm. This method was applied successfully to a magnetic suspension system in [4]. The convergence of the controller parameters to the solution of the correlation equation in the presence of noise and modeling errors was studied in [3].

In this paper, the fact that perfect decorrelation of the closed-loop output error and the reference signal is not possible (e.g. for the restricted-order controllers) is investigated. In this case, the two-norm of the correlation function is minimized using the extended instrumental variables method. Using the stochastic approximation method, it can be shown that the iterative algorithm converges to a local minimum. The frequency behavior of the resulting closed-loop system is compared with that of the initial one using the asymptotic frequency interpretation of the criterion. The analysis shows that, in the frequency regions where the additive uncertainties are not large, the achieved controller is close to the initial one, whilst in the frequency regions where the additive uncertainties are large, the gain of the achieved controller is reduced to improve the robustness of the system.

The paper is organized as follows. Section 2 presents the basics of the correlation

approach. An iterative procedure minimizing the correlation function is presented in Section 3. The frequency-domain analysis of the proposed method is given in Section 4. The theoretical results are illustrated via a simulation example in Section 5, and some concluding remarks are given in Section 6 .

\section{The Correlation Approach}

Figure 1 shows the block diagram for the model-following problem. The upper part presents the achieved closed-loop system and the lower part shows the designed closedloop system containing the initial model of the plant $\left(G_{0}\right)$ and the initial controller $\left(K_{0}\right)$. It is assumed that the initial controller is able to meet the specifications for the designed closed-loop system. 


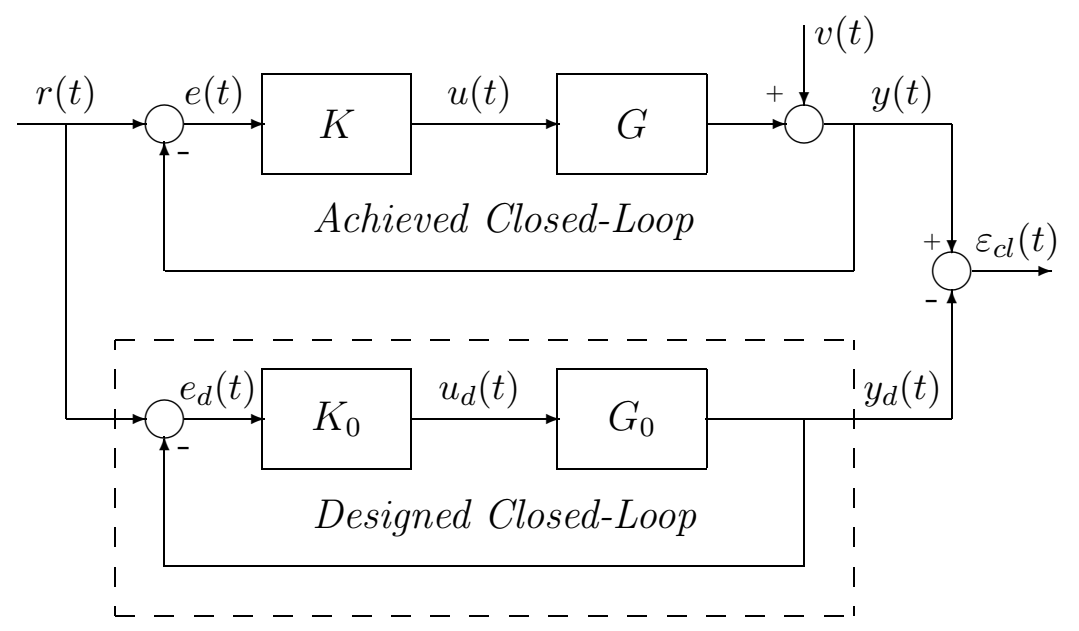

Figure 1: Block diagram of the achieved and designed closed-loop systems

Let the plant output be described as:

$$
y(t)=G\left(q^{-1}\right) u(t)+v(t)
$$

where $q^{-1}$ is the delay operator, $G\left(q^{-1}\right)$ an unknown LTI SISO discrete-time transfer operator, $u(t)$ the plant input and $v(t)$ a zero-mean stationary noise. The closed-loop output error is defined as:

$$
\varepsilon_{c l}(\rho, t)=y(\rho, t)-y_{d}(t)
$$

where $y(\rho, t)$ is the output of the achieved closed-loop system, $y_{d}(t)$ the output of the designed closed-loop system or the desired output, and $\rho$ the vector of controller parameters.

Let the initial controller $K_{0}$ be applied to the real system excited by the reference signal $r(t)$ and the plant output be measured. Then, the closed-loop output error contains the effect of both modeling errors and noise. Evidently, the effect of modeling errors is correlated with the reference signal, while that of noise is not. Since the lack of control performance results essentially from the modeling error, an improved controller should be able to compensate the effect of the modeling errors to the point that the closed-loop output error contains only filtered noise. Thus, a reasonable way to tune the controller parameters is to make the closed-loop output error independent of the reference signal. Let the correlation function $f(\rho)$ be defined as follows:

$$
f(\rho)=E\left\{\zeta(\rho, t) \varepsilon_{c l}(\rho, t)\right\}
$$

where $E\{\cdot\}$ is the mathematical expectation and $\zeta(\rho, t)$ a column vector of instrumental variables that are correlated with the reference signal and independent of the noise. The controller $K^{*}$ that leads to a perfect decorrelation $(f(\rho)=0)$ between the closed-loop output error and the reference signal is given below:

$$
K^{*}=K_{0} \frac{G_{0}}{G}
$$

However, this controller may be improper or of very high order, and therefore cannot be attained by the adopted controller structure. Thus, the tuning objective can be defined as the minimization of the following criterion:

$$
J(\rho)=\|f(\rho)\|_{2}^{2}=f^{T}(\rho) f(\rho)
$$


where $\|\cdot\|_{2}$ represents the two-norm. Then the controller parameter vector $\rho^{*}$ is given by:

$$
\rho^{*}=\arg \min _{\rho} J(\rho)
$$

Since this problem cannot be solved analytically, an iterative numerical method is considered. The advantage of the iterative algorithms based on gradient search is that inconvenient singular solutions are not attainable. For example, consider the case where the unstable zeros and poles of $G$ are not included in $G_{0}$ and the controller $K^{*}$ makes the closed-loop system internally unstable. Since this solution is a singular point on the criterion hyperplane, it cannot be approached with the iterative algorithm.

\section{Iterative tuning algorithm}

The controller parameter vector $\rho^{*}$ is solution to the gradient equation:

$$
J^{\prime}(\rho)=f^{T}(\rho) \frac{\partial f(\rho)}{\partial \rho}=0
$$

This problem can be approached by the Robbins-Monro [5] procedure using the following iterative formula:

$$
\rho_{i+1}=\rho_{i}-\gamma_{i}\left[Q\left(\rho_{i}\right)\right]^{-1}\left[J^{\prime}\left(\rho_{i}\right)\right]^{T}
$$

where $\gamma_{i}$ is a scalar step size and $Q\left(\rho_{i}\right)$ is a positive definite square matrix. This algorithm converges to a local minimum of the criterion provided that:

- $\sum_{i=1}^{\infty} \gamma_{i}=\infty, \quad \sum_{i=1}^{\infty} \gamma_{i}^{2}<\infty$

- The reference signal $r(t)$ and the disturbance signal $v(t)$ at each iteration are bounded independent stationary stochastic processes.

- The output of the closed-loop system $y(t)$ is bounded at each iteration (the closedloop system is stable).

The gradient of the criterion involves the expectations of signals that are unknown and should be replaced by their estimates using the closed-loop sampled data. Let the correlation function be estimated by $\bar{f}(\rho)$ :

$$
\bar{f}(\rho)=\frac{1}{N} \sum_{t=1}^{N} \zeta(\rho, t) \varepsilon_{c l}(\rho, t)
$$

where $N$ is the number of data. Then the derivative of the criterion is determined as follows:

$$
J^{\prime}\left(\rho_{i}\right)=\bar{f}^{T}\left(\rho_{i}\right) \frac{1}{N} \sum_{t=1}^{N}\left\{\left.\frac{\partial \zeta(\rho, t)}{\partial \rho}\right|_{\rho_{i}} \varepsilon_{c l}\left(\rho_{i}, t\right)+\left.\zeta\left(\rho_{i}, t\right) \frac{\partial \varepsilon_{c l}(\rho, t)}{\partial \rho}\right|_{\rho_{i}}\right\}
$$

An accurate value of the gradient cannot be computed because the derivative of $\varepsilon_{c l}(\rho, t)$ with respect to $\rho$ is unknown. However, an unbiased model-free estimation of this value can be obtained using two extra closed-loop experiments as is done in the IFT approach 
[2]. This value can also be estimated using the initial model of the plant or by a closedloop identified model using the same closed-loop data as the controller tuning [4]. In the latter case, the effects of the modeling errors on the convergence of the algorithm for different choices of the instrumental variables are studied in [3]. It is shown that the modeling errors do not affect the parametric convergence as long as a SPR condition on some transfer function is satisfied.

In order to improve the convergence speed, $Q\left(\rho_{i}\right)$ can be chosen as an approximation of the Hessian of the criterion (Gauss-Newton direction). In this case, one has:

$$
Q\left(\rho_{i}\right)=\left.\left(\left.\frac{\partial \bar{f}(\rho)}{\partial \rho}\right|_{\rho_{i}}\right)^{T} \frac{\partial \bar{f}(\rho)}{\partial \rho}\right|_{\rho_{i}}
$$

\section{Frequency-domain analysis}

In this section, the frequency characteristics of the achieved closed-loop system are compared with those of the designed closed-loop system. The relation between crosscorrelation functions and spectral density functions helps obtain an asymptotic equivalent of the criterion in the frequency domain.

For the simplicity of the analysis, the following choice of instrumental variables is considered:

$$
\zeta^{T}(t)=\left[r\left(t+n_{z}\right), r\left(t+n_{z}-1\right), \ldots, r(t), r(t-1), \ldots, r\left(t-n_{z}\right)\right]
$$

where $n_{z}$ is a sufficiently large integer number. Thus, the criterion of Eq. 5 can be presented as:

$$
J(\rho)=f^{T}(\rho) f(\rho)=\sum_{\tau=-n_{z}}^{n_{z}} R_{\varepsilon r}^{2}(\tau)
$$

where $R_{\varepsilon r}(\tau)$ is the cross-correlation function between the reference signal $r(t)$ and the closed-loop output error $\varepsilon_{c l}(t)$ defined by:

$$
R_{\varepsilon r}(\tau)=E\left\{\varepsilon_{c l}(\rho, t) r(t-\tau)\right\}
$$

From Fig. 1, the closed-loop output error can be expressed as:

$$
\varepsilon_{c l}(\rho, t)=\left(T\left(q^{-1}, \rho\right)-T_{0}\left(q^{-1}\right)\right) r(t)+S\left(q^{-1}, \rho\right) v(t)
$$

where $S\left(q^{-1}, \rho\right)$ and $T\left(q^{-1}, \rho\right)$ are the sensitivity function and the complementary sensitivity function of the achieved closed-loop system, respectively, and $T_{0}\left(q^{-1}\right)$ is the designed complementary sensitivity function defined as follows $\left(q^{-1}\right.$ is omitted):

$$
S(\rho)=\frac{1}{1+K G} \quad, \quad T(\rho)=\frac{K G}{1+K G} \quad, \quad T_{0}=\frac{K_{0} G_{0}}{1+K_{0} G_{0}}
$$

Then, with the assumption that $v(t)$ and $r(t)$ are not correlated, one obtains:

$$
R_{\varepsilon r}(\tau)=E\{H(\rho) r(t) r(t-\tau)\}=\sum_{i=0}^{\infty} h(i) R_{r r}(\tau-i)
$$


where $H(\rho)=T(\rho)-T_{0}, h(t)$ is its impulse response, and $R_{r r}(\tau)$ the auto-correlation function of $r(t)$. On the other hand, $R_{\varepsilon r}(\tau)$ can be expressed as an integral in the frequency domain [7]:

$$
R_{\varepsilon r}(\tau)=\frac{1}{2 \pi} \int_{-\pi}^{\pi} H\left(e^{-j \omega}, \rho\right) \Phi_{r}(\omega) e^{j \tau \omega} d \omega
$$

where $\Phi_{r}(\omega)$ is the spectrum of the reference signal $r(t)$. Now, replacing $R_{\varepsilon r}(\tau)$ in the criterion of Eq. 13 by the expressions from Eqs 16 and 17 gives:

$$
\begin{aligned}
J(\rho) & =\sum_{\tau=-n_{z}}^{n_{z}}\left(\sum_{i=0}^{\infty} h(i) R_{r r}(\tau-i)\right)\left(\frac{1}{2 \pi} \int_{-\pi}^{\pi} H\left(e^{-j \omega}, \rho\right) \Phi_{r}(\omega) e^{j \tau \omega} d \omega\right) \\
& =\sum_{\tau=-n_{z}}^{n_{z}} \frac{1}{2 \pi} \int_{-\pi}^{\pi} \sum_{i=0}^{\infty} h(i) e^{i j \omega} R_{r r}(\tau-i) e^{j(\tau-i) \omega} H\left(e^{-j \omega}, \rho\right) \Phi_{r}(\omega) d \omega \\
& =\int_{-\pi}^{\pi} \sum_{i=0}^{\infty} h(i) e^{i j \omega}\left(\frac{1}{2 \pi} \sum_{\tau=-n_{z}}^{n_{z}} R_{r r}(\tau-i) e^{j(\tau-i) \omega}\right) H\left(e^{-j \omega}, \rho\right) \Phi_{r}(\omega) d \omega
\end{aligned}
$$

Therefore, when $n_{z}$ tends to infinity, using the symmetrical property of the autocorrelation functions $\left(R_{r r}(\tau)=R_{r r}(-\tau)\right)$, one obtains:

$$
\lim _{n_{z} \rightarrow \infty} J(\rho)=\int_{-\pi}^{\pi}\left|H\left(e^{-j \omega}, \rho\right)\right|^{2} \Phi_{r}^{2}(\omega) d \omega
$$

It is interesting to compare this criterion with that of the closed-loop output error minimization in the frequency domain. Using the expression of $\varepsilon_{c l}(\rho, t)$ in 15 , straightforward calculations give:

$$
J_{\varepsilon}(\rho)=R_{\varepsilon \varepsilon}(0)=\frac{1}{2 \pi} \int_{-\pi}^{\pi}\left[\left|H\left(e^{-j \omega}, \rho\right)\right|^{2} \Phi_{r}(\omega)+\left|S\left(e^{-j \omega}, \rho\right)\right|^{2} \Phi_{v}(\omega)\right] d \omega
$$

This criterion shows that there is a trade off between noise attenuation (via the sensitivity function $S\left(e^{-j \omega}, \rho\right)$ ) and model following (via $H\left(e^{-j \omega}, \rho\right)$ ). One can see clearly that the criterion based on the correlation approach Eq. 21 is not influenced by the noise signal $v(t)$ and that the spectral density of the reference signal is emphasized with a power of two in the criterion.

If the reference signal is white noise with variance 1 , and $n_{z}$ tends to infinity, one has:

$$
\begin{aligned}
\rho^{*} & =\arg \min _{\rho} \int_{-\pi}^{\pi}\left|T\left(e^{-j \omega}, \rho\right)-T_{0}\left(e^{-j \omega}\right)\right|^{2} d \omega=\arg \min _{\rho} \int_{-\pi}^{\pi}\left|S\left(e^{-j \omega}, \rho\right)-S_{0}\left(e^{-j \omega}\right)\right|^{2} d \omega \\
& =\arg \min _{\rho} \int_{-\pi}^{\pi}\left|S\left(e^{-j \omega}, \rho\right)\right|^{2}\left|K G-K_{0} G_{0}\right|^{2}\left|S_{0}\left(e^{-j \omega}\right)\right|^{2} d \omega
\end{aligned}
$$

where $S_{0}=\left(1+K_{0} G_{0}\right)^{-1}$ is the designed sensitivity function. These relations show that the achieved complementary sensitivity function $T\left(e^{-j \omega}, \rho\right)$ and, consequently, the achieved sensitivity function $S\left(e^{-j \omega}, \rho\right)$ tend to their respective designed functions. Thus, the tuned controller ensures the designed performance in tracking and disturbance rejection for the real closed-loop system (robust performance). It can also be seen that the open-loop gain of the real system $K G$ will be close to the designed one $K_{0} G_{0}$ in the frequencies where the magnitude of the sensitivity function is high.

Now, consider the effect of the tuned controller on the input sensitivity function $U(\rho)=$ $K S(\rho)$. For this purpose, Eq. 23 is rearranged as follows (the arguments are omitted):

$$
\rho^{*}=\arg \min _{\rho} \int_{-\pi}^{\pi}|S|^{2}\left|G_{0}\left(K-K_{0}\right)+K\left(G-G_{0}\right)\right|^{2}\left|S_{0}\right|^{2} d \omega
$$


Consider the frequency regions where $G_{0}$ is small but the additive uncertainty $G-G_{0}$ is large (the middle and high frequencies). In these regions, the algorithm tries to minimize $|U(\rho)|^{2}\left|G-G_{0}\right|^{2}\left|S_{0}\right|^{2}$. Since $S_{0}$ is near to 1 at high frequencies, the amplitude of the input sensitivity function $U(\rho)$ is reduced where the additive uncertainties are large (robust stability). The robustness properties of the proposed tuning method are illustrated via a simulation example in the next section.

\section{Simulation example}

The system $G(s)=B(s) / A(s)$, taken from [6], with

$$
\begin{aligned}
B(s)= & 6.599 \cdot 10^{-5} s^{9}-2.552 \cdot 10^{-3} s^{8}-0.1264 s^{7} \\
& -0.2836 s^{6}-4.195 s^{5}+6.983 s^{4}-13.74 s^{3} \\
& +215.2 s^{2}+144.0 s+1057 \\
A(s)= & s^{9}+2.401 s^{8}+32.68 s^{7}+54.78 s^{5}+347.2 s^{5} \\
& +347.2 s^{5}+351.2 s^{4}+1256 s^{3} \\
& +488.8 s^{2}+635.3 s+105.9
\end{aligned}
$$

is considered. The system is discretized with the sampling period $T_{s}=\pi / 8$. The initial controller is calculated by pole placement using the following identified 4th-order model

$$
G_{0}(s)=\frac{B_{0}\left(q^{-1}\right)}{A_{0}\left(q^{-1}\right)}=\frac{-4.51 \cdot 10^{-4} q^{-1}+0.0218 q^{-2}+0.0378 q^{-3}+0.0152 q^{-4}}{1-2.721 q^{-1}+2.516 q^{-2}-0.751 q^{-3}-0.0366 q^{-4}}
$$

The log-magnitude Bode plots of the system $G$ and the model $G_{0}$ are drawn in Fig. 2 . The curves corresponding to $G$ and $G_{0}$ show quite a good match around the first resonant mode at $0.765 \mathrm{rad} / \mathrm{s}$, whereas there is a large discrepancy around the second resonant mode at $3.45 \mathrm{rad} / \mathrm{s}$.

The design objective is to damp the dominant oscillatory modes of the open-loop system, but to preserve their natural frequencies. The controller contains two fixed terms. Firstly, to ensure a correct static gain and to suppress low frequency disturbances, an integrator $R_{f i x}=1-q^{-1}$ is included in the polynomial $R$. Secondly, to avoid noise amplification and control signal saturation a factor $S_{f i x}=1+0.975 q^{-1}$ is included in the polynomial $S$, which reduces the controller gain close to the Nyquist frequency. The resulting 5th-order initial controller $K_{0}\left(q^{-1}\right)=S_{0}\left(q^{-1}\right) / R_{0}\left(q^{-1}\right)$ is given as follows:

$$
K_{0}\left(q^{-1}\right)=\frac{\left(1+0.975 q^{-1}\right)\left(0.4177-1.4736 q^{-1}+1.6283 q^{-2}-0.5154 q^{-3}-0.0560 q^{-4}\right)}{\left(1-q^{-1}\right)\left(1-0.2841 q^{-1}+0.2301 q^{-2}+0.0521 q^{-3}+0.0138 q^{-4}\right)}
$$

A 7th-order controller $K$ is to be tuned on the real system. Note that the order of the optimal controller $K^{*}$ that would perfectly decorrelate the output error $\varepsilon_{c l}$ with the instrumental variables $\zeta$ is 18 (there is no zero-pole cancellation between the transfer functions $G$ and $K_{0} \cdot G_{0}$ in Eq. 4). The instrumental variable vector is chosen as in Eq. 12 with $n_{z}=39$.

The tuning procedure is carried out in 8 iterations, with each being performed using a different realization of the noise $v(t)$ that provides a noise/signal ratio of about $7.5 \%$ in terms of variance. The reference signal is a PRBS generated by a 7-bit shift register with data length $N=2048$. In all iterations, the constant step size $\gamma_{i}=0.5$ is used. 


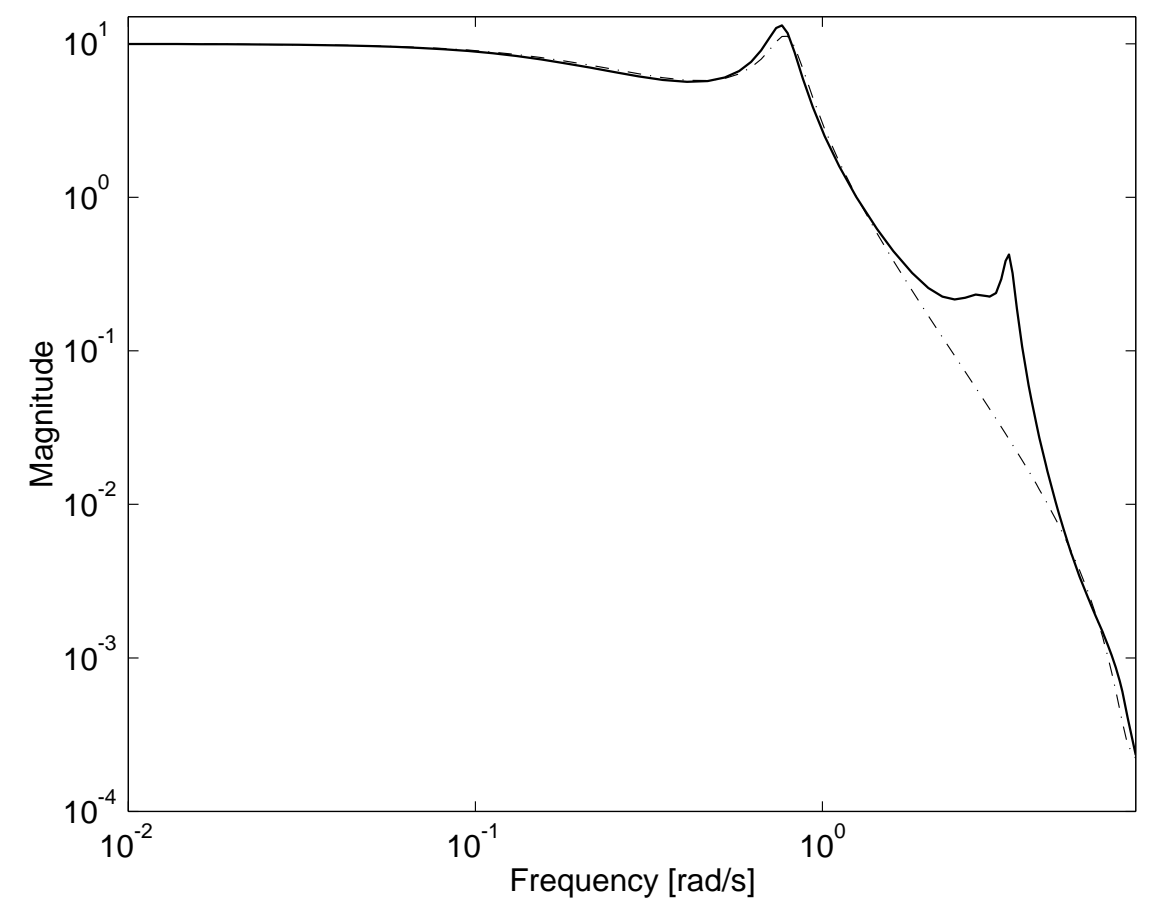

Figure 2: Bode plots of $G$ (solid) and $G_{0}$ (dash-dot)

Fig. 3 shows the complementary sensitivity functions $T_{0}, T_{i n}=K_{0} G\left(1+K_{0} G\right)^{-1}$ and $T$ for the designed, initial and final closed-loop systems, respectively. It can be seen that, though the obtained controller $K$ reduces the peak of the sensitivity function around 3.45 $\mathrm{rad} / \mathrm{s}$, it does not suppress it completely. This can be explained by the fact that the order of the controller is not sufficient for perfect decorrelation.

Fig. 4 depicts the corresponding sensitivity functions $S_{0}, S_{i n}=\left(1+K_{0} G\right)^{-1}$ and $S$. A comparison of the curves shows great similarity of the designed and resulting sensitivity functions. This leads to the conclusion that the resulting closed-loop system exhibits robust performance.

The input sensitivity functions $U_{i n}=K S_{i n}$ and $U$ are given in Fig. 5 together with that of the designed model $U_{0}=K_{0} S_{0}$. These curves clearly show that the resulting controller $K$ reduces the sensitivity function $U$ at the frequencies where the model uncertainty is large (around $3.45 \mathrm{rad} / \mathrm{s}$ ), thus trying to improve robustness.

\section{Conclusions}

A new criterion for controller tuning based on the correlation approach is proposed. The criterion is defined as the two-norm of the cross-correlation function between the closedloop output error and the reference signal. With the assumption that the reference signal and the noise are independent, the criterion is asymptotically not affected by the noise characteristics. The frequency-domain analysis of the proposed criterion shows that the difference between the achieved and designed closed-loop systems weighted by the square of the spectrum of the reference signal is minimized by the algorithm. Therefore, the designed closed-loop performance in terms of both tracking and disturbance rejection is preserved for the achieved closed-loop system. In addition, the input sensitivity function 


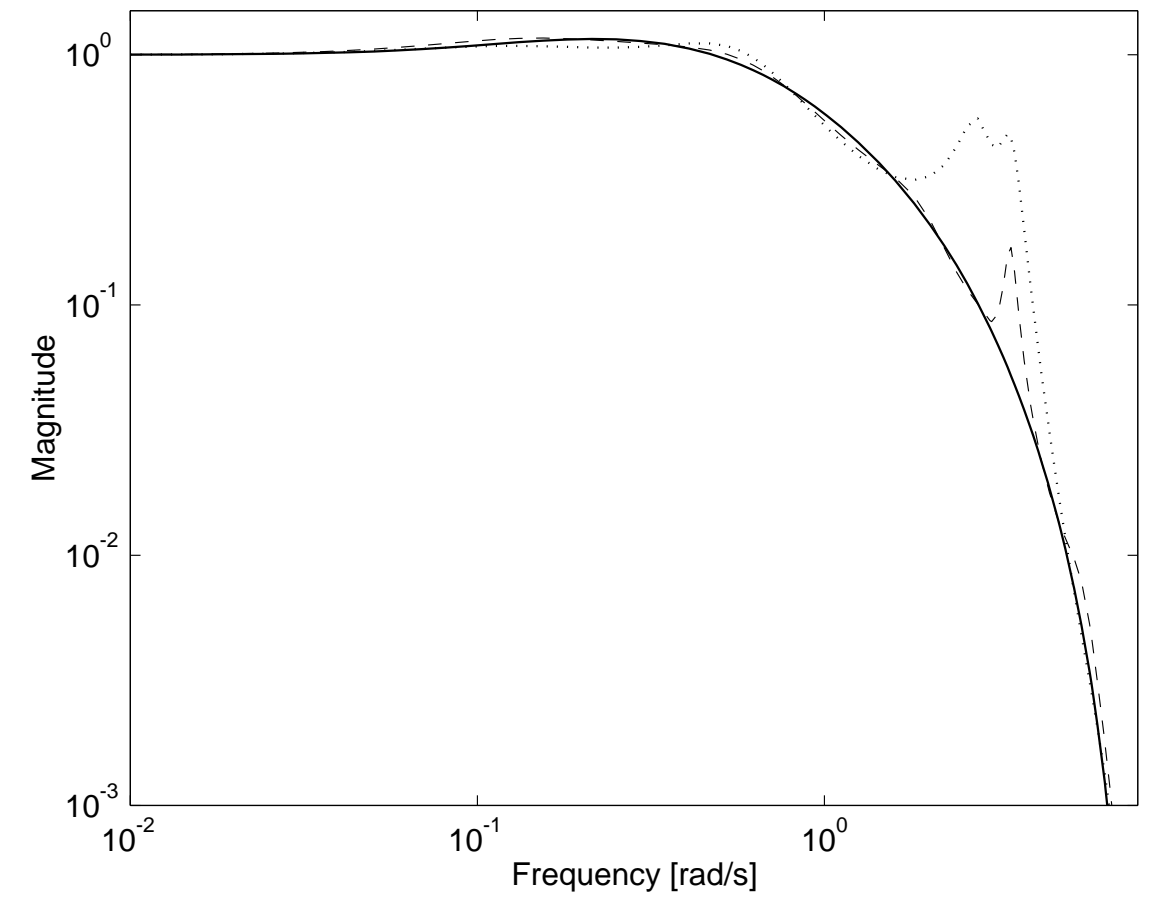

Figure 3: Magnitude plots of the sensitivity functions $T_{0}$ (solid), $T_{\text {in }}$ (dotted) and $T$ (dashed)

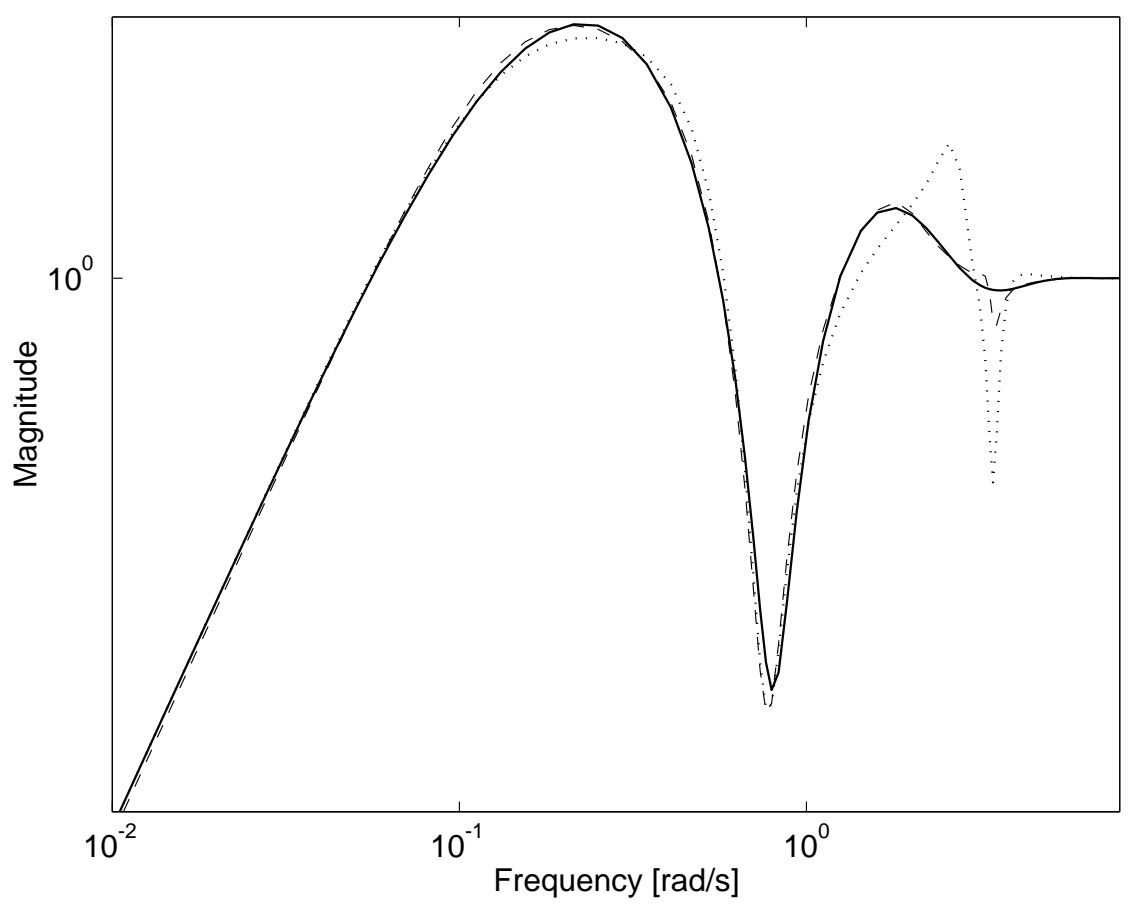

Figure 4: Magnitude plots of the sensitivity functions $S_{0}$ (solid), $S_{\text {in }}$ (dotted) and $S$ (dashed)

is decreased in the frequency regions where the additive uncertainty is large. Hence, a robust controller can be tuned using only closed-loop data with no information regarding model uncertainties. 


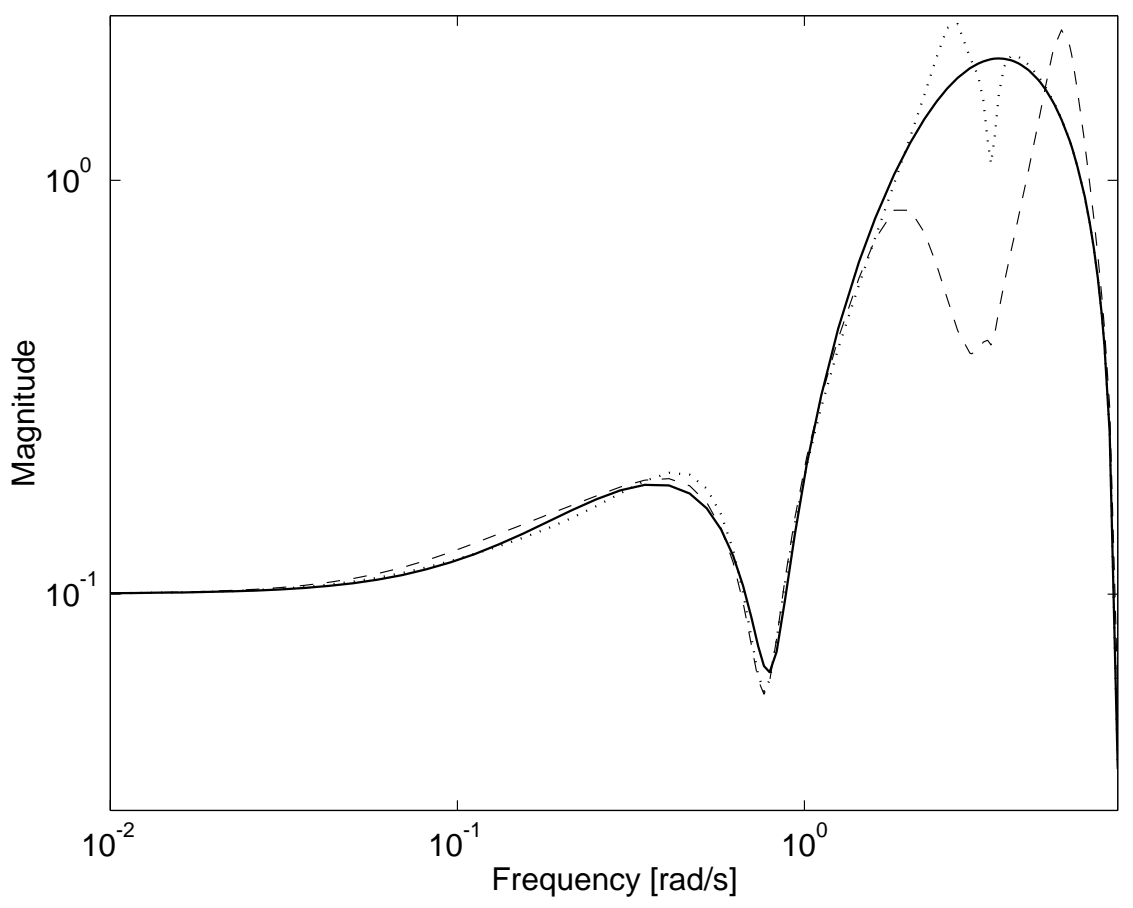

Figure 5: Magnitude plots of the sensitivity functions $U_{0}$ (solid), $U_{\text {in }}$ (dotted) and $U$ (dashed)

\section{References}

[1] K. J. Åström and B. Wittenmark. Adaptive Control. Addison-Wesley, 1989.

[2] H. Hjalmarsson, S. Gunnarsson, and M. Gevers. A convergent iterative restricted complexity control design scheme. In 33rd IEEE-CDC, volume 2, pages 1735-1740, December 1994.

[3] A. Karimi, L. Mišković, and D. Bonvin. Convergence analysis of an iterative correlation-based controller tuning method. In 15th IFAC World Congress, Barcelona, Spain, July 2002.

[4] A. Karimi, L. Mišković, and D. Bonvin. Iterative controller tuning based on the correlation approach. To be published in Control Engineering Practice, 2002.

[5] H. Robbins and S. Monro. A stochastic approximation method. Ann. Math. Stat., 22:400-407, 1951.

[6] R. R. Schrama. Accurate identification for control: The necessity of an iterative scheme. IEEE Transactions on Automatic Control, 37(7):991-994, July 1990.

[7] T. Söderström and P. Stoica. System Identification. Prentice-Hall, U.K., 1989.

[8] E. Trulsson and L. Ljung. Adaptive control based on explicit criterion minimization. Automatica, 21(4):385-399, 1985. 